\title{
Control de inventario y sistema de distribución en una cooperativa agraria
}

Inventory control and distribution system in an agricultural cooperative

Controle de estoque e sistema de distribuição em uma cooperativa agropecuária

\author{
Nieves del Pilar Pizzán-Tomanguillo \\ nievesdelpilarpizzan1993@gmail.com \\ CNL ASESORES - Perú \\ ORCID: https://orcid.org/0000-0001-9933-8738
}

José Alfredo Reátegui Salas

jars.reateguisalas@gmail.com

CNL ASESORES - Perú

ORCID: https://orcid.org/0000-0001-6255-1201

Carlos Daniel Rosales Bardalez

bardalez.daniel@gmail.com

Universidad Peruana Unión - Perú

ORCID: https://orcid.org/0000-0002-2311-1577

\begin{abstract}
RESUMEN
El presente estudio tuvo como objetivo determinar de qué manera el control de inventario se relaciona con el sistema de distribución de la Cooperativa Agraria El Gran Saposoa, 2021, Se empleó un tipo de investigación aplicada, diseño no experimental, de corte transversal, de nivel descriptivo correlacional. Para la recolección de datos se determinó una población de 113 colaboradores y como muestra a 56, se aplicó la técnica de encuesta y como instrumento el cuestionario. Para la presentación de los resultados se empleó un análisis descriptivo e inferencial, que revelaron que el control de inventario presenta un nivel regular en un 64\% de la misma manera el sistema de distribución en un $61 \%$. Por lo tanto, se concluye que el control de inventario se relaciona de manera directa y significativa con el sistema de distribución de la Cooperativa Agraria El Gran Saposoa, en vista que se obtuvo un p-valor=0.00<0.05, y un coeficiente de correlación de R de Pearson positiva fuerte de 0.824. Esto indica, que al presentar el control de inventario un comportamiento creciente, el sistema de distribución tiende a crecer. Del mismo modo, el tamaño del efecto demostró que el control de inventario influye un $67.8 \%$ en el sistema de distribución.
\end{abstract}

Palabras clave: Control de inventario, Sistema de distribución, Compras, Recepción, Almacenamiento.

\begin{abstract}
The objective of this study was to determine how inventory control is related to the distribution system of the El Gran Saposoa Agrarian Cooperative, 2021. A type of applied research, non-experimental design, cross-sectional, descriptive level was used. correlational. For data collection, a population of 113 collaborators was determined and 56 as a sample, the survey technique was applied and the questionnaire as an instrument. For the presentation of the results, a descriptive and inferential analysis was used, which revealed that the inventory control presents a regular level in $64 \%$, in the same way the distribution system in $61 \%$. Therefore, it is concluded that inventory control is directly and significantly related to the distribution system of the El Gran Saposoa Agrarian Cooperative, given that a pvalue $=0.00<0.05$ was obtained, and a correlation coefficient strong positive Pearson's $\mathrm{R}$ of 0.824 . This indicates that when inventory control presents an increasing behavior, the distribution system tends to grow. Similarly, the effect size showed that inventory control influences $67.8 \%$ in the distribution system.
\end{abstract}

Keywords: Inventory control, Distribution system, Purchases, Reception, Storage.

\section{RESUMO}

O objetivo deste estudo foi determinar como o controle de estoque está relacionado ao sistema de distribuição da Cooperativa Agrária El Gran Saposoa, 2021. Foi utilizado um tipo de pesquisa aplicada, desenho não experimental, transversal, nível descritivo. Para a coleta de dados foi determinada uma população de 113 colaboradores e 56 como 
amostra, foi aplicada a técnica de survey e o questionário como instrumento. Para a apresentação dos resultados foi utilizada uma análise descritiva e inferencial, que revelou que o controle de estoque apresenta um nível regular em $64 \%$, da mesma forma que o sistema de distribuição em 61\%. Portanto, conclui-se que o controle de estoque está direta e significativamente relacionado ao sistema de distribuição da Cooperativa Agrária El Gran Saposoa, dado que se obteve um valor de $\mathrm{p}=0,00<0,05$, e um coeficiente de correlação forte $\mathrm{R}$ de Pearson positivo de 0,824 . Isso indica que quando o controle de estoque apresenta um comportamento crescente, o sistema de distribuição tende a crescer. Da mesma forma, o tamanho do efeito mostrou que o controle de estoque influencia $67,8 \%$ no sistema de distribuição.

Palavras-chave: Controle de Estoque, Sistema de Distribuição, Compras, Recepção, Armazenagem.

\section{INTRODUCCIÓN}

Las empresas agroindustriales y comerciales tienen como base fundamental el control eficiente de sus inventarios, para ello, adoptan técnicas, y métodos asertivos, así también, registros que garanticen el monitoreo minucioso de los productos, por otro lado, el personal encargado de dichas funciones son pilares para la coordinación y supervisión de todos los procesos, el conocimiento y experiencia que presenten garantizará el funcionamiento adecuado dentro de las áreas que involucran el control de inventario. Sin embargo, en su mayoría, las empresas ejecutan sus actividades de manera empírica, con la finalidad de minimizar gastos, pero los resultados pueden afectar a la producción y al cliente final.

Tal es el caso, de las empresas ecuatorianas, donde el artículo expuesto por González (2019) expresa que el $46 \%$ de las empresas presentan el incumplimiento de normas y lineamientos que limita un adecuado control del inventario, el $22 \%$ no cuentan con el personal capacitado y con los conocimientos necesarios para dirigir actividades de control y monitoreo. Y el 32\%, tienen dificultades en el empleo y registro de papeletas de control, permitiendo faltantes, deterioros, extravíos y sobreproducción de productos. Todos estos hechos afectan de manera directa a la distribución de los productos, por cuanto existe retrasos, errores en la entrega de mercadería y devoluciones por parte de los clientes.

En el Perú, se ha registrado 395 cooperativas agrarias, el 90\% de ellas son micro y pequeñas empresas, el $77 \%$ cuenta con un gerente y el 58\% de ellos, presentan estudios superiores (Ministerio de la Producción, 2020). En la actualidad estas empresas han generado 17 mil puestos de trabajos, debido a las funciones que demanda su actividad y están integradas en su mayoría por productores con conocimiento básicos, sin embargo, presentan limitantes en las actividades que demanda conocimientos técnicos y metodológicos.

Por consiguiente, Ccoicca (2020) alude en su artículo que estas organizaciones presentan falencias en la asistencia técnica de sus actividades, asimismo la información especificada en los registros carece de veracidad y asertividad. El 55\% de ellas, incumple con el tiempo determinado para su distribución, generando insatisfacción en los clientes que buscan obtener sus productos de manera oportuna, estos sucesos provocaron que el sistema de distribución logístico de dichas organizaciones se vea afectado.

La problemática brevemente descrita, no es ajena a las empresas agrarias locales, por cuanto presentan falencias semejantes a lo antes mencionado, como es el caso de la Cooperativa Agraria El Gran Saposoa, cuya actividad es la producción y comercialización de granos de cacao y café, y cuenta con 5 áreas (abastecimiento, logística, almacén, distribución y transporte, ventas) que dirigen la comercialización de los productos, el incumplimiento o falencias que pueda presentarse en una de estas áreas, puede ocasionar la desorganización de las actividades y pérdida de los productos, es por ello que es necesario el seguimiento continuo y oportuno de los mismos.

Sin embargo, con los resultados obtenidos por la organización, se ha puesto en evidencia las falencias relacionados al control del inventario, como la falta de interés en la aplicación de la NIC 2, que describe el tratamiento de las existencias, dado que el control de la misma se desarrolla de manera empírica, generando que los saldos de valor de inventarios sean incorrectos e irreales, por lo demás se observa desorden en el almacenamiento de productos, presentando desmedros del 
producto, y hasta pérdida de los mismos. Así también, los controles son desarrollados parcialmente y discontinuos, y las herramientas aplicadas no son las apropiadas para el monitoreo del producto, dado que los formatos o papeletas de control no contienen la información pertinente para determinar decisiones que convienen a la organización.

En cuanto, a las compras se realizan sin la programación y planificación debida, por lo que en situaciones se desconoce la cantidad de la materia prima y suministros. Por consiguiente, no existe una distribución adecuada y oportuna de los productos solicitados, dado que durante el transporte se deterioran y no se cumple con la fecha y hora de la entrega del producto, lo que conlleva a la pérdida de la cartera de cliente.

Es por ello, que se formula el problema general: ¿De qué manera el control de inventario se relaciona con el sistema de distribución de la Cooperativa Agraria El Gran Saposoa, 2021? Problemas específicos: ¿De qué manera las compras se relacionan con el sistema de distribución de la Cooperativa Agraria El Gran Saposoa, 2021?; ¿De qué manera la recepción se relaciona con el sistema de distribución de la Cooperativa Agraria El Gran Saposoa, 2021? y ¿De qué manera el almacenamiento se relaciona con el sistema de distribución de la Cooperativa Agraria El Gran Saposoa, 2021?

El objetivo general que persigue la investigación es: Determinar de qué manera el control de inventario se relaciona con el sistema de distribución de la Cooperativa Agraria El Gran Saposoa, 2021. Objetivos específicos: Determinar de qué manera las compras se relacionan con el sistema de distribución de la Cooperativa Agraria El Gran Saposoa, 2021, Determinar de qué manera la recepción se relaciona con el sistema de distribución de la Cooperativa Agraria El Gran Saposoa, 2021 y Determinar de qué manera el almacenamiento se relaciona con el sistema de distribución de la Cooperativa Agraria El Gran Saposoa, 2021.

El trabajo de investigación se justifica, bajo un valor teórico, porque en la investigación se describió características y comportamiento de las variables, haciendo uso de teorías e investigaciones relacionadas al control de inventario y distribución. También presenta relevancia social, porque el resultado alcanzado será de beneficio a las empresas que presenten realidades semejantes, en el diseño y reestructuración de los procesos de control de inventario y distribución, a fin de que puedan cumplir con los objetivos empresariales planteados. La justificación práctica del estudio recae en la importancia de mostrar las acciones negativas realizadas por la Cooperativa, y se espera que los responsables tomen conciencia sobre la situación actual, y determinen si es necesario la implementación de un sistema de control. Desde un contexto metodológico, se hará el uso de técnicas, procedimientos e instrumentos para la recolección de resultados cercanos a la realidad, dejando a un lado la razón del investigador y desarrollando de manera asertiva los objetivos planteados en la investigación

La hipótesis que responde el problema general es: Hi: El control de inventario se relaciona de manera directa y significativa con el sistema de distribución de la Cooperativa Agraria El Gran Saposoa, 2021. Ho: El control de inventario no se relaciona de manera directa y significativa con el sistema de distribución de la Cooperativa Agraria El Gran Saposoa, 2021.Hipótesis específicas: Hi1: Las compras se relacionan de manera significativa con el sistema de distribución de la Cooperativa Agraria El Gran Saposoa, 2021.Hi2: La recepción se relaciona de manera significativa con el sistema de distribución de la Cooperativa Agraria El Gran Saposoa, 2021. Hi3: El almacenamiento se relaciona de manera significativa con el sistema de distribución de la Cooperativa Agraria El Gran Saposoa, 2021.

En la investigación se desarrolla en el apartado dos el fundamento teórico, en la que se muestra las investigaciones relacionadas a las variables en estudio, como también la base teórica de las variables y dimensiones, por otro lado en el apartado tres, se desarrolla los procedimientos metodológicos, donde se describe la metodología utilizada, las técnicas e instrumentos aplicados en la investigación, en el apartado cuatro, se desarrollan los resultados y discusiones, se presenta los resultados en forma narrativa y grafica de acuerdo a los objetivos 
planteados, se realiza un análisis crítico sobre las mismas, para posteriormente comparar los mismos con otros estudios similares, sigue las conclusiones, se presenta en forma resumida la respuesta al objetivo del estudio, como se legró a lo que se llegó, también mostrar las limitaciones y sugerir futuras investigaciones, por último se muestra las referencias utilizadas en el desarrollo del artículo.

\section{FUNDAMENTO TEÓRICO}

\subsection{Antecedentes}

El trabajo de investigación se sustenta bajo estudios efectuados con anterioridad, las mismas que muestran realidades relacionadas a las variables. Como es el caso de Juca y Narváez (2019) quienes en su investigación concluyeron que la empresa Modesto Casajoana de Ecuador no cuenta con un control de existencias adecuado porque el procedimiento empleado evidencia falencias, no existe un grado óptimo de inventarios, no son suficientes para cubrir con los requerimientos de pedidos, ocasionando una pérdida monetaria de $\$ 27,395.68$. Así también, el control de las existencias se asocia de manera significativa en la cadena de suministros, puesto que el coeficiente estadístico de Rho Spearman fue de 0.000 y el grado de correlación alcanzó un valor de 0.802 .

De igual modo, Quinde \& Ramos (2018) en su artículo concluyeron que el 52\% de las organizaciones de Ecuador mantienen una administración de mercadería de manera particular, el cual impide una distribución eficiente de la mercadería, además, la organización no planifica a tiempo las futuras adquisiciones según las necesidades de esta. Y el control de existencias de la organización posee una conexión negativa con los resultados económicos debido a que la significancia fue inferior a 0.005, es decir, 0.000, lo cual indica que existe relación entre ambos.

Asimismo, Tenesca \& Maji (2018) concluyeron que el $75 \%$ de las empresas agrarias presentan un control deficiente, esto se debe a que cualquier persona puede acceder a la mercadería, no existe una comunicación eficiente, no existe planificación ni seguimiento de la mercadería, los registros Kardex no se encuentran debidamente actualizados. Como también, el control de inventarios se relaciona con los beneficios económicos de la organización, visto que obtuvo una significancia estadística de Pearson de 0.001, es decir, existe asociación entre ambos temas estudiados.

Por otro lado, Díaz \& Cedeño (2017) señalan que el control de inventarios en las empresas de Guayaquil - Ecuador es poco eficiente, porque la distribución de mercadería no es desarrollada de forma adecuada, el espacio del almacén es pequeño para conservar toda la mercadería, tampoco tienen conocimiento exacto del presupuesto para las futuras adquisiciones y desconocen del producto que mayor rotación genera en la empresa. Con respecto a la asociación entre la gestión de inventarios y la decisión financiera, esta es significativa con un resultado estadístico de 0.000 , el cual señala que el control de existencias influye en la toma de decisiones.

También Iturrizaga (2017) en su informe de investigación concluyó que en la Distribuidora Leal SAC no existe una distribución adecuada de la mercadería porque existe dificultad para la entrega de cada producto terminado, asimismo genera el aumento de los costos el cual ha ocasionado considerables pérdidas monetarias, así como la pérdida de clientes. Además, se evidenció que existe vínculo entre las variables con un valor de 0.000 y un resultado de correlación de 0.759 . Existe asociación entre la administración de almacén y distribución con una significancia de 0.000 y una correlación de 0.845 .

Por su lado, Sánchez (2017) en su investigación que trata de gestión de almacén y distribución física en el área de logística y producción de la empresa Gloria S.A, donde concluyo que la distribución en la organización es desarrollada de forma regular, dado que no realizan la inspección correspondiente de cada producto, no existe un registro oportuno detallado sobre los 
productos que sufrieron daños en el almacén y no existe información del producto de mayor rotación en la organización. Por consiguiente, los procesos de recepción son inadecuada, dado que los productos registrados en los documentos no guardan relación con los comprobantes, así también no se verifica las cantidades y cualidades, por lo tanto, existe relación directa con la distribución de los productos, generando un nivel de significancia de 0.00 y una correlación de Rho de Spearman de 0.784 .

De igual manera, López (2019) en su estudio concluyó que existe relación entre la gestión de control y el manejo de inventario, dado que el coeficiente de Rho Spearman resultó 0.000 y una correlación de 0.793 , es decir las variables inciden en un $63 \%$.

\subsection{Control de inventario}

Según, Oluwaseyi (2017) define que es una actividad que implica la supervisión de las existencias que posee una compañía para mantener el balance correcto de los inventarios en el almacén Para Meana (2017), son actividades de compras, recepción y almacenamiento, su eficiencia parte de los recursos materiales y humanos que participan en control y seguimientos de las actividades, así también del cumplimiento de las acciones previstas en cada uno de los procesos. Sus dimensiones son las siguientes:

Como primera dimensión tenemos a compras, Meana (2017) sostiene que es una de las actividades que inicia con la información de las cantidades o niveles de existencias habidas en almacén, cuando los niveles son mínimos o no abastecen las áreas productivas, es necesario efectuar una compra, para ello es importante contar con proveedores que garanticen la entrega de los productos a buena calidad y precio. Los indicadores son: constancia de valoración, análisis y selección de la propuesta, emisión de la solicitud de compra.

En cuanto, a la dimensión recepción, Meana (2017), sostiene que en este proceso el responsable (jefe de almacenamiento) realiza las coordinaciones pertinentes para recibir el producto, empleando los registros que permitan la evaluación y seguimiento del producto en cuanto a calidad y cantidad, a fin de evitar irregularidad que se presentan al momento de la venta. Cabe mencionar, que todo ingreso debe ser respaldado por documentos, donde se visualice de manera clara y precisa, las cantidades exactas, y, por consiguiente, deben ser guardados en lugares apropiadas para su respectiva sustentación. Los indicadores son: estado de las materias y suministros, verificación de cantidad, elaboración de ingreso a bodega:

Con respecto a la dimensión almacenamiento, Meana (2017), en este proceso se realiza el ingreso de los productos, ubicándolo según la línea de comercialización, tamaño, así también se realiza el respectivo etiquetamiento del producto, a fin de facilitar su ubicación durante la comercialización del producto. El responsable, debe realizar ciertas actividades de control y supervisión de manera continua, para evitar el deterioro y el vencimiento del producto, y por ende generar pérdidas que pueden afectar las ventas y rendimiento económico de la organización. Los indicadores son: clasificación de los productos, codificación de los productos, registro en el sistema de inventario saldos

\subsection{Sistema de distribución}

Escudero (2019) fundamenta que la distribución es un conjunto de procesos por la cual una organización traslada y distribuye los materiales que fueron adquiridas de un destino a otro de forma eficaz, proporcionando de esta manera satisfacción a la demanda de los clientes. En cuanto, Hart (2017) son actividades relacionadas y ligadas al área de ventas, las actividades entre ellas, debe ser coordinada y programada oportunamente, para evitar la aparición de acciones incorrectas durante la distribución. Al mismo tiempo que Dybskaya \& Sverchkov (2017) refieren que es una 
acción de contexto empresarial que tiene como propósito elaborar planes y administrar las actividades vinculadas con el flujo óptimo de los productos.

Las dimensiones de la variable son: procesamiento de los pedidos, según Escudero (2019), corresponde a todas las actividades en función a la recepción, comprobación y transmisión de las órdenes de compra. Cuando llega un pedido a las instalaciones se genera un conjunto de actividades que se desarrollan en cadena, hasta que los productos se trasladen a las unidades de transporte para su respectivo envío al cliente. El almacenamiento debe contar con una serie de lineamiento, que sancionen actividades incorrectas, de esa manera limitar entregas a destiempo, o productos deteriorados durante el transporte. Tiene los siguientes indicadores: verificación del orden de pedido y preparación del pedido

En cuanto a la dimensión transporte, consiste en la preparación de los materiales para su transporte correspondiente hasta el cliente. En este proceso, debe existir una relación unida y fortalecida entre el área de ventas y los transportistas, de esa manera coordinar adecuadamente el lugar de entrega, la verificación de los documentos que se entregarán al cliente y formalidad. Es importante que la Cooperativa adopte protocolos de entregas, para lograr la satisfacción del cliente y el compromiso de la empresa, estos protocolos, deben ser diseñados de acuerdo con la realidad y situación. Tiene los siguientes indicadores: agrupar los pedidos y trasladar los pedidos

Finalmente, en la dimensión entrega de mercadería, son todas las acciones para la entrega de mercadería al destino final. En este apartado, es importante la aplicación de protocolos y se debe realizar la entrega de la documentación al cliente, para acreditar la entrega del producto, posterior a ello, se debe entregar al área correspondiente de su evaluación (contabilidad). Tiene los siguientes indicadores: verificación de comprobantes y actualización del nivel de stock

\section{PROCEDIMIENTOS METODOLOGICOS}

\subsection{Tipo y diseño}

La investigación presenta un enfoque cuantitativo, de tipo aplicada, en vista que Rivero (2018) sostiene que se caracteriza por la aplicación de teorías e investigaciones para enriquecer y fortalecer los resultados alcanzados, generando a través de ello, conocimientos concretos y consistente, empleando métodos estadísticos para dar solución a las hipótesis planteadas en el estudio.

Así también, presenta un diseño no experimental de corte transversal, según Quintana et al. (2017) este se limita a la manipulación de los resultados, no obstante, se analizan y evalúan los datos según su comportamiento en su contexto natural en un periodo dado, para ello se efectúan procedimientos y técnicas que faciliten la obtención de resultados fehacientes.

\subsection{Población y muestra}

La población estuvo comprendida por 113 colaboradores de la Cooperativa Agraria El Gran Saposoa. En los criterios de inclusión se ha considerado a los empleados del área de abastecimiento, logística, almacén, transporte y de ventas. En la investigación se ha limitado aquellos que no decidieron participar voluntariamente, entre ellos colaboradores del área administrativa, área comercial, contable y especialistas del producto.

De acuerdo, a las necesidades de la investigación y a los objetivos planteados, se ha determinado como muestra a 56 colaboradores. Según Alarcón (2016), la muestra es un subconjunto de la población que presentan características y comportamientos similares (p.32) 
Se aplicó un muestreo no probabilístico, por conveniencia, según Arbaiza (2019), la selección de los elementos depende de la intención propia del investigador, limitando la aplicación de fórmulas o medios estadísticos. En el estudio, únicamente se ha encuestado a 56 colaboradores de las áreas de abastecimiento, logística, almacén, distribución y transporte, ventas, para estimar los procesos de compras, recepción, almacenamiento y distribución.

\subsection{Técnica e instrumento}

De acuerdo con los objetivos planteados, fue necesario aplicar como técnica la encuesta. Según, Navas (2017). es una técnica aplicada a investigaciones descriptivas, es decir, que buscan conocer a detalle las características, cualidades y comportamiento de las variables. En el estudio, fue pertinente aplicar la encuesta, dado que se busca relacionar las variables, así también conocer a detalle las características del control de inventario y el sistema de distribución.

El instrumento aplicado fue el cuestionario, el mismo que fue estructurado según las dimensiones e indicadores que componen a las variables (control de inventario y el sistema de distribución). El cuestionario de la variable control de inventario y sistema de distribución cuentan con 19 enunciados. La escala de Likert aplicado fue: nunca; casi nunca; a veces; casi siempre y siempre.

Para el cálculo del coeficiente de confiabilidad, se ha empleado el procesamiento estadístico SPSS 28. De acuerdo con el instrumento de la variable control interno, se obtuvo un coeficiente de alfa de Cronbach de 0.854, demostrando una alta confiabilidad para la aplicación de este. Así también del instrumento de la variable sistema de distribución se obtuvo un coeficiente de 0.868 , por lo que se procedió a su aplicación a los 56 colaboradores de la Cooperativa Agraria El Gran Saposoa.

\subsection{Procedimiento y análisis de datos}

Uno de las actividades principales fue solicitar la autorización del gerente de la Cooperativa Agraria El Gran Saposoa, a fin de poder aplicar los instrumentos elaborados, tras la aceptación se ha determinado una reunión con el personal seleccionado en la muestra (56 colaboradores), ya con los cuestionarios llenados, se ha separado los resultados según su categorización, mediante codificaciones, siendo estos tabulados en hojas Excel, así también se ha empleado procesamiento estadístico SPSS, para resolver los objetivos inferenciales.

Según la estructura de los objetivos planteados en el estudio, se aplicaron métodos y procedimientos que se ajustan a la investigación, entre ellos el análisis descriptivo, según Navarro, y Jiménez (2017), este análisis consiste en captar la caracterización y cualidades de los elementos en estudio, demostrando tendencias en relación con la escala de medición. En ese sentido, en la investigación se expuso el nivel descriptivo de las variables, detallando las puntuaciones obtenidas a través de frecuencias y porcentajes. También, se aplicó el análisis inferencial, que tuvo como fin la obtención de conclusiones y probar hipótesis planteados, para ello será necesario emplear la prueba de normalidad, con la finalidad de determinar la prueba paramétrica a emplear ( $\mathrm{R}$ Pearson) por consiguiente aceptar o rechazar la hipótesis planteada. Además, se analizará el coeficiente de correlación, mediante los siguientes niveles:

En la investigación se ha empleado los principios de beneficencia dado que en el desarrollo del informe se ha respetado los derechos y valores de los encuestados, para ello, fue importante conocer la participación voluntaria de los mismos. Así también, con los resultados alcanzados, se pretende beneficiar a la empresa, a fin de que puedan corregir y adoptar procesos razonables para un mejor control de los productos. Además, se aplicó el principio de autonomía, por cuanto, para la 
aplicación de los instrumentos, fue importante contar con la autorización del gerente de la Cooperativa Agraria El Gran Saposoa, así también el consentimiento de los colaboradores.

\section{RESULTADOS Y DISCUSIÓN}

\subsection{Resultados descriptivos}

\section{Tabla 1}

Análisis descriptivo del control de inventarios y sus dimensiones

\begin{tabular}{|c|c|c|c|c|c|c|c|c|}
\hline \multirow[t]{2}{*}{ Variable/Dimen. } & \multicolumn{2}{|c|}{$\begin{array}{l}\text { Control de } \\
\text { inventario }\end{array}$} & \multicolumn{2}{|c|}{ Compras } & \multicolumn{2}{|c|}{ Recepción } & \multicolumn{2}{|c|}{ Almacenamiento } \\
\hline & $\mathrm{f}$ & $\%$ & $\mathrm{f}$ & $\%$ & $\mathrm{f}$ & $\%$ & $\mathrm{f}$ & $\%$ \\
\hline Inadecuado & 13 & $23 \%$ & 19 & $34 \%$ & 11 & $20 \%$ & 20 & $36 \%$ \\
\hline Regular & 36 & $64 \%$ & 29 & $52 \%$ & 37 & $66 \%$ & 26 & $46 \%$ \\
\hline Adecuado & 7 & $13 \%$ & 8 & $14 \%$ & 8 & $14 \%$ & 10 & $18 \%$ \\
\hline Total & 56 & $100 \%$ & 56 & $100 \%$ & 56 & $100 \%$ & 56 & $100 \%$ \\
\hline
\end{tabular}

El control de inventario presenta un nivel regular en un 64\%, debido al incumplimiento de las actividades que permiten un adecuado monitoreo de las materias adquiridas para la producción.

Por lo que refiere al proceso de compra es regular en un 52\%, dado que las cotizaciones son seleccionadas de manera incorrecta, sin el análisis correcto para un precio justo de los productos, por consiguiente, el incumplimiento de las entregas atrasa la producción y hasta la fecha no se ha sancionado a los proveedores por tal acto. De igual manera el proceso de recepción se desarrolla de manera regular en un $66 \%$, dado que la verificación de las cantidades y calidad de la materia no se verifica al momento de adquirirlas, lo que ha generado insuficiencia en la elaboración de los productos, sin embargo, existe un informe donde se detalla de manera específica la situación del caso.

Así también, el proceso de almacenamiento es regular en un 46\%, dado que no se realza la clasificación correcta de las materias, según sus características, así también, algunos de ellos no cuentan con el etiquetamiento correspondiente que facilite la producción de los productos. No obstantes los datos expuestos a la asociación se acercan a la realidad del caso y cuentan con veracidad. Los resultados se relacionan con el estudio realizado por Quinde \& Ramos (2018) quienes concluyeron que el 52\% de las organizaciones de Ecuador mantienen una administración de mercadería de manera particular, el cual impide una distribución eficiente de la mercadería, además, la organización no planifica a tiempo las futuras adquisiciones según las necesidades de esta.

\section{Tabla 2}

Análisis descriptivo del sistema de distribución y sus dimensiones

\begin{tabular}{lcccccccc}
\hline \multicolumn{1}{c}{ Variable/Dimen. } & \multicolumn{2}{c}{$\begin{array}{c}\text { Sistema de } \\
\text { distribución }\end{array}$} & \multicolumn{2}{c}{$\begin{array}{c}\text { Procesamiento de } \\
\text { pedidos }\end{array}$} & \multicolumn{2}{c}{ Transporte } & \multicolumn{2}{c}{$\begin{array}{c}\text { Entrega de } \\
\text { pedidos }\end{array}$} \\
& $\mathrm{F}$ & $\%$ & $\mathrm{~F}$ & $\%$ & $\mathrm{~F}$ & $\%$ & $\mathrm{~F}$ & $\%$ \\
\hline Inadecuado & 14 & $25 \%$ & 17 & $30 \%$ & 11 & $20 \%$ & 13 & $23 \%$ \\
Regular & 34 & $61 \%$ & 25 & $45 \%$ & 23 & $41 \%$ & 36 & $64 \%$ \\
Adecuado & 8 & $14 \%$ & 14 & $25 \%$ & 22 & $39 \%$ & 7 & $13 \%$ \\
Total & 56 & $100 \%$ & 56 & $100 \%$ & 56 & $100 \%$ & 56 & $100 \%$ \\
\hline
\end{tabular}

Los resultados obtenidos demuestran que el sistema de distribución presenta un nivel regular del $61 \%$, así también los procesos de procesamiento de pedidos en un $45 \%$, debido a que existen problemas en la revisión de las órdenes de pedidos, que en casos no contienen de manera detallada 
las características, cantidades y costos, pero es importante mencionar que contienen las firmas correspondientes para la salida y aprobación del producto.

En cuanto a los procesos de transporte, también presenta un nivel regular del $41 \%$, con tendencia a un nivel alto de 39\%, dado que la selección de los pedidos es agrupada según ordenes de pedidos, así también para el transportista cuenta con toda la documentación y detalles para evitar equivocaciones, sin embargo, es importante señalar que no se priorizan los pedidos con urgencia, lo que causa disgustos en los clientes.

Finalmente, en el proceso de entrega de los productos, también es percibido de nivel regular en un $64 \%$ dado que los incumplimientos de las actividades dirigidas al transportista no son sancionados, dando paso a constantes deficiencias, así también existe problemas en la actualización de data, tras la entrega de los productos, lo que causa desconocimiento de las cantidades de las materias almacenadas.

También el estudio desarrollado por Díaz \& Cedeño (2017) presenta resultados semejantes dado que concluyeron que las empresas de Guayaquil - Ecuador la distribución de mercadería no es desarrollada de forma adecuada, el espacio del almacén es pequeño para conservar toda la mercadería, tampoco tienen conocimiento exacto del presupuesto para las futuras adquisiciones y desconocen del producto que mayor rotación genera en la empresa

\subsection{Resultados inferenciales}

Con la finalidad de verificar la distribución normal de las variables, así también la prueba paramétrica a emplear, se procede aplicar la siguiente prueba:

Tabla 3

Prueba de normalidad

\begin{tabular}{llll}
\hline & $\begin{array}{l}\text { Kolmogorov-Smirnov } \\
\text { Estadístico }\end{array}$ & gl & Sig. \\
\hline V1. Control de inventario & 0.094 & 56 &, $200^{*}$ \\
V2. Sistema de distribución & 0.089 & 56 &, $200^{*}$ \\
D1. Compras & 0.168 & 56 &, 051 \\
D2. Recepción & 0.124 & 56 &, 062 \\
D3. Almacenamiento & 0.205 & 56 &, 049 \\
\hline
\end{tabular}

De acuerdo con la prueba de normalidad de Kolmogorov-Smirnov, para muestras mayores a 50, los datos provenientes de las variables control de inventario y sistema de distribución, proceden de una distribución normal, por cuanto el p-valor obtenido $(0,200)$ es mayor al 0.050 , por lo tanto, se emplea la prueba paramétrica $r$ de Pearson para comprobar las hipótesis planteadas en la investigación.

\section{Hipótesis general}

Hi: El control de inventario se relaciona de manera significativa con el sistema de distribución de la Cooperativa Agraria El Gran Saposoa, 2021.

Ho: El control de inventario no se relaciona con el sistema de distribución de la Cooperativa Agraria El Gran Saposoa, 2021. 


\section{Tabla 4}

Relación entre el control de inventario y sistema de distribución

\begin{tabular}{rlrr}
\hline & & $\begin{array}{c}\text { Control de } \\
\text { inventario }\end{array}$ & $\begin{array}{c}\text { Sistema de } \\
\text { distribución }\end{array}$ \\
\hline \multirow{3}{*}{ Control de inventario } & Correlación de Pearson & 1 & $824^{* *}$ \\
& Sig. (bilateral) & 56 & 0.000 \\
& N &, $824^{* *}$ & 56 \\
& Correlación de Pearson & 0.000 & 1 \\
Sistema de distribución & Sig. (bilateral) & 56 & 56 \\
& $\mathrm{~N}$ & & \\
\hline
\end{tabular}

**. La correlación es significativa en el nivel 0,01 (bilateral).

Según la tabla 4 , muestra que existe relación estadísticamente significativa entre el control de inventario y el sistema de distribución, dado que el (p-valor=<0.05). Así también, el coeficiente de correlación de $\mathrm{R}$ de Pearson demostró una relación positiva fuerte de 0.824 , por lo tanto, se rechaza la hipótesis nula y se acepta la hipótesis que señala que "El control de inventario se relaciona de manera significativa con el sistema de distribución de la Cooperativa Agraria El Gran Saposoa, 2021". Por consiguiente, el control de inventario al demostrar una tendencia creciente, el sistema de distribución presentará una tendencia igual.

Por consiguiente, es importante hacer cálculo del tamaño del efecto, para ello, se emplea el coeficiente de correlación de Pearson elevado al cuadrado (0.824)2, cuyo resultado indica que el control de inventario influye un $67.8 \%$ en el sistema de distribución, y la diferencia (32\%) se debe a otros factores independientes al control de inventario. Esto demuestra, que la eficiencia o el cumplimiento de las actividades del control de inventario tienen un efecto en el asertividad del sistema de distribución, por lo que es importante desarrollarlo con la importancia del caso, para no afectar la materia prima disponible para la elaboración oportuna de los productos determinados y por ende el rendimiento económico de la organización.

De igual manera, Iturrizaga (2017) concluye que en la Distribuidora Leal SAC existe una distribución inadecuada de las mercaderías, por cuanto existe dificultades en la entrega de los productos, asimismo se genera aumentos de los costos el cual ha ocasionado considerables pérdidas monetarias, por lo que señala que existe vínculo entre la administración del almacén y distribución, dado que el p-valor fue de 0.000 y un coeficiente de correlación de RHO Spearman de 0.759. Además. Juca y Narváez (2019), sostienen que los procesos presentados en el control de inventario, como la falta de organización y verificación de las existencias incidieron en los requerimientos de pedidos, ocasionando considerables pérdidas monetarias. Asimismo, Quinde y Ramos (2018) concluye en su investigación que las actividades de organización de las mercaderías la cual impide una distribución eficiente y oportuna, dado que no se planifican las adquisiciones según las necesidades de las empresas ecuatorianas.

\section{Hipótesis específicas 1}

Hi: Las compras se relacionan de manera significativa con el sistema de distribución de la Cooperativa Agraria El Gran Saposoa, 2021.

Ho: Las compras no se relacionan con el sistema de distribución de la Cooperativa Agraria El Gran Saposoa, 2021.

Tabla 5

Relación entre compras y sistema de distribución 


\begin{tabular}{clrr}
\hline & & Compras & \multicolumn{2}{c}{$\begin{array}{c}\text { Sistema de } \\
\text { distribución }\end{array}$} \\
\hline \multirow{3}{*}{ Compras } & Correlación de Pearson & 1 &, $951^{* *}$ \\
& Sig. (bilateral) & & 0.000 \\
& N & 56 & 56 \\
& Correlación de Pearson &, $951^{* *}$ & 1 \\
Sistema de distribución & Sig. (bilateral) & 0.000 & 56 \\
& $\mathrm{~N}$ & 56 & 5 \\
\hline
\end{tabular}

**. La correlación es significativa en el nivel 0,01 (bilateral).

De igual manera la tabla 5, demuestra que existe relación estadísticamente significativa entre las compras y el sistema de distribución, dado que el (p-valor=<0.05). Así también, el coeficiente de correlación de $\mathrm{R}$ de Pearson demostró una relación positiva fuerte de 0.951 , por lo tanto, se rechaza la hipótesis nula y se acepta la hipótesis que señala que "Las compras se relacionan de manera significativa con el sistema de distribución de la Cooperativa Agraria El Gran Saposoa, 2021. Por consiguiente, el proceso de compras al demostrar una tendencia creciente, el sistema de distribución presentará una tendencia igual.

Asimismo, el estudio desarrollado por López (2019) sostiene que la empresa Inversiones Shimba, no existe un eficiente análisis de las necesidades de compra, por lo que se ha presentado un sobre stock de los productos y en casos el desabastecimiento del mismo, esto tuvo como consecuencias afectación en los beneficios económicos de la empresa, por ello sostiene que existe relación entre la gestión de control y el manejo de inventario, dado que obtuvo un nivel de significancia menor al 0.05 y el coeficiente de Rho Spearman resultó de 0.793

\section{Hipótesis específicas 2}

Hi: La recepción se relaciona de manera significativa con el sistema de distribución de la Cooperativa Agraria El Gran Saposoa, 2021.

Ho: La recepción no se relaciona con el sistema de distribución de la Cooperativa Agraria El Gran Saposoa, 2021.

Tabla 6

Relación entre recepción y sistema de distribución

\begin{tabular}{clrr}
\hline & & Recepción & \multicolumn{2}{c}{$\begin{array}{c}\text { Sistema de } \\
\text { distribución }\end{array}$} \\
\hline \multirow{2}{*}{ Recepción } & Correlación de Pearson & 1 &, $793^{* *}$ \\
& Sig. (bilateral) & 0.000 \\
& N & 56 & 56 \\
Sistema de distribución & Correlación de Pearson &, $793^{* *}$ & 1 \\
& Sig. (bilateral) & 0.000 & 56 \\
\hline
\end{tabular}

**. La correlación es significativa en el nivel 0,01 (bilateral).

Así también, la tabla 6, demuestra que existe relación estadísticamente significativa entre la recepción y el sistema de distribución, dado que el ( $\mathrm{p}$-valor=<0.05). Así también, el coeficiente de correlación de R de Pearson demostró una relación positiva fuerte de 0.793 , por lo tanto, se rechaza la hipótesis nula y se acepta la hipótesis que señala que "La recepción se relaciona de manera significativa con el sistema de distribución de la Cooperativa Agraria El Gran Saposoa, 2021". Por consiguiente, el proceso de recepción al demostrar una tendencia creciente, el sistema de distribución presentará una tendencia igual. 
Los resultados guardan relación con lo obtenidos por Sánchez (2017), dado que concluye que los procesos de recepción son desarrollados de manera inadecuada, dado que los productos registrados en los documentos no guardan relación con los comprobantes, así también no se verifica las cantidades y cualidades, es por ello que existe relación directa con la distribución de los productos, generando un nivel de significancia de 0.00 y una correlación de Rho de Spearman de 0.784 .

\section{Hipótesis específicas 3}

Hi: El almacenamiento se relaciona de manera significativa con el sistema de distribución de la Cooperativa Agraria El Gran Saposoa, 2021

Ho: El almacenamiento no se relaciona con el sistema de distribución de la Cooperativa Agraria El Gran Saposoa, 2021.

Tabla 7

Relación entre almacenamiento y sistema de distribución

\begin{tabular}{clrr}
\hline & & Almacenamiento & $\begin{array}{c}\text { Sistema de } \\
\text { distribución }\end{array}$ \\
\hline \multirow{3}{*}{ Almacenamiento } & Correlación de Pearson & 1 &, $350^{* * *}$ \\
& Sig. (bilateral) & 0.008 \\
& N & 56 & 56 \\
& Correlación de Pearson &, $350^{* *}$ & 1 \\
& Sig. (bilateral) & 0.008 & 56 \\
& $\mathrm{~N}$ & 56 & 56 \\
\hline
\end{tabular}

**. La correlación es significativa en el nivel 0,01 (bilateral).

Así también, la tabla 7, demuestra que existe relación estadísticamente significativa entre el almacenamiento y el sistema de distribución, dado que el ( $p$-valor $=<0.05$ ). Así también, el coeficiente de correlación de $\mathrm{R}$ de Pearson demostró una relación positiva débil de 0.350 , por lo tanto, se rechaza la hipótesis nula y se acepta la hipótesis que señala que "El almacenamiento se relaciona de manera significativa con el sistema de distribución de la Cooperativa Agraria El Gran Saposoa, 2021". Por consiguiente, el proceso de almacenamiento al demostrar una tendencia creciente, el sistema de distribución presentará una tendencia igual.

También, el estudio desarrollado por Iturrizaga (2017) concluye que existe asociación entre la administración de almacén de la Distribuidora Leal SAC y los procesos de distribución, por cuanto existe un nivel de significancia de 0.000 y una correlación de Rho Spearman de 0.845. Además, Sánchez (2017) concluye en su investigación que la distribución desarrollada por la empresa Gloria S.A es regular, dado que no realizan la inspección correspondiente de cada producto, no existe un registro oportuno detallado sobre los productos que sufrieron daños en el almacén y no existe información del producto de mayor rotación en la organización.

\section{CONCLUSIÓN}

Tras un análisis inferencial se ha definido que el control de inventario se relaciona de manera directa y significativa con el sistema de distribución de la Cooperativa Agraria El Gran Saposoa, en vista que se obtuvo un $\mathrm{p}$-valor $=0.00<0.05$, y un coeficiente de correlación de $\mathrm{R}$ de Pearson positiva fuerte de 0.824. Esto indica, que al presentar el control de inventario un comportamiento creciente, el sistema de distribución tiende a crecer. Del mismo modo, el tamaño del efecto demostró que el control de inventario influye un $67.8 \%$ en el sistema de distribución. Cabe mencionar que las dimensiones del control de inventario como las compras, recepción y almacenamiento también se encuentran relacionadas de manera significativa con la distribución, de 
modo que es importante la estimación continua de los procesos para obtener resultados que impacten de manera positiva en el cumplimiento de los objetivos institucionales.

En lo que respecta a las limitaciones presentadas en el estudio, la muestra no fue suficientemente representativa para lograr recolectar más información, es decir, es necesario considerar a los jefes de las áreas determinadas en el estudio, en vista que tienen más información y conocimiento de los procedimientos enfocados al control de inventarios y distribución.

A raíz de los resultados, como agenda futura de investigación se presentan las siguientes interrogantes: ¿Cuáles son los cambios presentados en el control de inventario tras la aparición de la pandemia en la Región de San Martín?, ¿Cuáles son los procesos más eficaces en la distribución de productos en las empresas agrarias de la Región de San Martín?

\section{REFERENCIAS}

Alarcón, R. (2016). Métodos y Diseños de Investigación del Comportamiento. (2ed). Universidad Ricardo Palma. Editorial Universitaria.

Arbaiza, L. (2019). Como elaborar una tesis de grado. Esan Ediciones

Ccoicca, K. (2020). Control de inventario en la distribución logística en la empresa Ransa Comercial S.A., Callao, 2020. Universidad Cesar Vallejo. Lima - Perú. https://cutt.ly/ezCKzLW

Díaz, A. y Cedeño, M. (2017). Gestión de inventarios y su incidencia en la toma de decisiones financieras. Observatorio de la Economía Latinoamericana. 3 (16), 1-7. https://cutt.ly/3zOIha8

Escudero, M. (2019). Logística de Almacenamiento. ( $2^{\circ}$ ed.). Ediciones Paraninfo. https://cutt.ly/tzPKLx7

González, R. (2019). Control de inventarios y su incidencia en la rentabilidad de la empresa Codilitesa S.A. Ambato Ecuador. https://cutt.ly/PzCmk0K

Iturrizaga, O. (2017). Gestión de almacén y distribución en la Distribuidora Leal SAC, Juanjui, San Martin, 2017. (Tesis de pregrado). Universidad Cesar Vallejo. Lima - Perú. https://cutt.ly/XzOKNMV

Juca, C. y Narváez, C. (2019). Modelo de gestión y control de inventarios para la determinación de los niveles óptimos en la cadena de suministros de la Empresa Modesto Casajoana Cía. Ltda. Revista Digital Publisher. 4 (3), 1939. https://cutt.ly/MzOyRBx

López, E. (2019). Gestión de control y su relación con el manejo de inventario de la empresa Inversiones Shimba, Tarapoto año 2018. (Tesis de pregrado). Universidad Cesar Vallejo. San Martín - Perú. https://cutt.ly/EzPRVyF

Meana, P. (2017) Gestión de inventario. ( $\left.1^{\circ} \mathrm{ed}\right)$. Ediciones Nobel

Ministerio de la Producción (2020), Primer Censo Nacional de Cooperativas: https://agraria.pe/noticias/existen-395cooperativas-agrarias-en-el-peru-15298

Navarro, E. y Jimenez, E. (2017) Fundamentos de la investigación y la innovación educativa. ( $1^{\circ}$ ed). Unir editorial

Navas, V. (2017). Metodología de la investigación social. ( $\left.1^{\circ} \mathrm{ed}\right)$. Lemoine Editores y Editorial Delgado.

Oluwaseyi, J. (2017). Evaluation of the Role of Inventory Management in Logistics Chain of an Organisation. LOGIScientific Journal on Transport and Logistics. 8 (2), 1-11. https://cutt.ly/dzAylif

Quinde, C. y Ramos, T. (2018). Valuación y control del inventario y su efecto en la rentabilidad. Revista Observatorio de la Economía Latinoamericana. 3 (11), 1-12. https://cutt.ly/lzOlobc

Quintana, J., García, B., Riesco, M., Fernández, E. y Sánchez, J. (2018). Fundamentos básicos de metodología de investigación educativa. ( $\left.3^{\circ} \mathrm{ed}\right)$. Ediciones de la U.

Rivero, D. (2018). Metodología de la investigación. (3ed). Editorial Shalom

Sánchez, T. (2017). Gestión de almacén y distribución física en el área de logística y producción de la empresa Gloria S.A. en Huachipa - lima 2017. (Tesis de pregrado). Universidad Cesar Vallejo. Lima - Perú. https://cutt.ly/UzO95CA

Tenesca, M. y Maji, E. (2018). Control de los inventarios y su incidencia en la rentabilidad para negocios que comercializan productos de primera necesidad. Observatorio de la Economía Latinoamericana. 4 (11). 1-10. https://cutt.ly/GzOngXZ 